\title{
Photocatalysis: new highlights from JEP 2013
}

\author{
Sylvie Lacombe • Fernando Fresno • \\ Urška Lavrenčič Štangar
}

Received: 28 May 2014 / Accepted: 10 June 2014 / Published online: 21 June 2014

(C) Springer-Verlag Berlin Heidelberg 2014

The scientific and technological implications of photocatalytic processes are well recognized today, insofar as they allow the possibility of performing chemical reactions aimed at the elimination of pollutants, the inactivation of bacteria, the production of fuels and the synthesis of high added value chemicals without the concourse of elevated temperatures and pressures and using (sun)light as the only energy input.

Heterogeneous photocatalysis is based on the promotion of electrons from the filled valence band (VB) to the empty conduction band (CB) of a semiconductor when it is irradiated with light with photon energy equal to or greater than the energy gap between those two levels (band gap energy, $\mathrm{E}_{\mathrm{g}}$ ), leaving positively charged holes behind. These photogenerated electrons and holes can migrate to the surface of the solid and there react with adsorbed electron acceptors and donors, respectively, thus catalysing reduction-oxidation reactions. Based on this premise, different applications of heterogeneous photocatalysis emerge.

Among these applications, the degradation of pollutants from water and air has historically been, and remains, the most researched one (Fig. 1) and is indeed the only one, together with the chemically related inactivation of bacteria

Responsible editor: Philippe Garrigues

S. Lacombe

Institute for Analytical Sciences and Physico-Chemistry for Environment and Materials (IPREM), UMR 5254 CNRS, University of Pau and Pays de l'Adour, Hélioparc, 2 avenue of President ANGOT, 64053 Pau Cedex 09, France

e-mail: sylvie.lacombe@univ-pau.fr

F. Fresno $(\bowtie) \cdot$ U. Lavrenčič Štangar

Laboratory for Environmental Research, University of Nova Gorica,

Vipavska 13, 5000 Nova Gorica, Slovenia

e-mail: fernando.fresno@ung.si

U. Lavrenčič Štangar

e-mail: urska.lavrencic@ung.si and self-cleaning surfaces, that has found a market niche up to date. In the presence of organic compounds adsorbed on the photocatalyst surface, these and any subsequent intermediates may be oxidized in several steps until mineralization to carbon dioxide, water and inorganic acids by direct reaction with holes or by highly oxidizing intermediates generated from them. Although there is still some debate concerning the mechanisms of hole trapping, it has been generally accepted that water species adsorbed on terminal $\mathrm{Ti}$ atoms trap the holes forming hydroxyl radicals. Therefore, heterogeneous photocatalysis can be framed into the so-called advanced oxidation processes (AOPs), aimed at the mineralization of pollutants through the mediation of $\mathrm{OH}$ radicals. In turn, the reduction of oxidized species (invariably oxygen in environmental applications, although the co-participation of other oxidizing reactants like ozone or hydrogen peroxide may increase the rate of the process) by conduction band electrons further generates oxidizing intermediates like $\mathrm{O}_{2}{ }^{-}$and $\mathrm{OOH}$ before eventually being reduced to water.

The possible environmental applications of heterogeneous photocatalysis may be summarized as follows:

- Air treatment: purification of outdoor and indoor air and off-gas emissions, elimination of odours, ethylene elimination during the storage of fruits and vegetables, soil remediation by air stripping and subsequent photocatalytic treatment, etc.

- Water treatment: wastewater detoxification for biological treatment, discolouration, rendering water suitable for human consumption or for agricultural or industrial use, etc.

- Active surfaces: self-cleaning, self-sterilizing, selfdegrading or anti-fogging materials (metals, ceramics 
Fig. 1 Number of research articles published in the last 10 years related to the main applications of heterogeneous photocatalysis. Source: Scopus

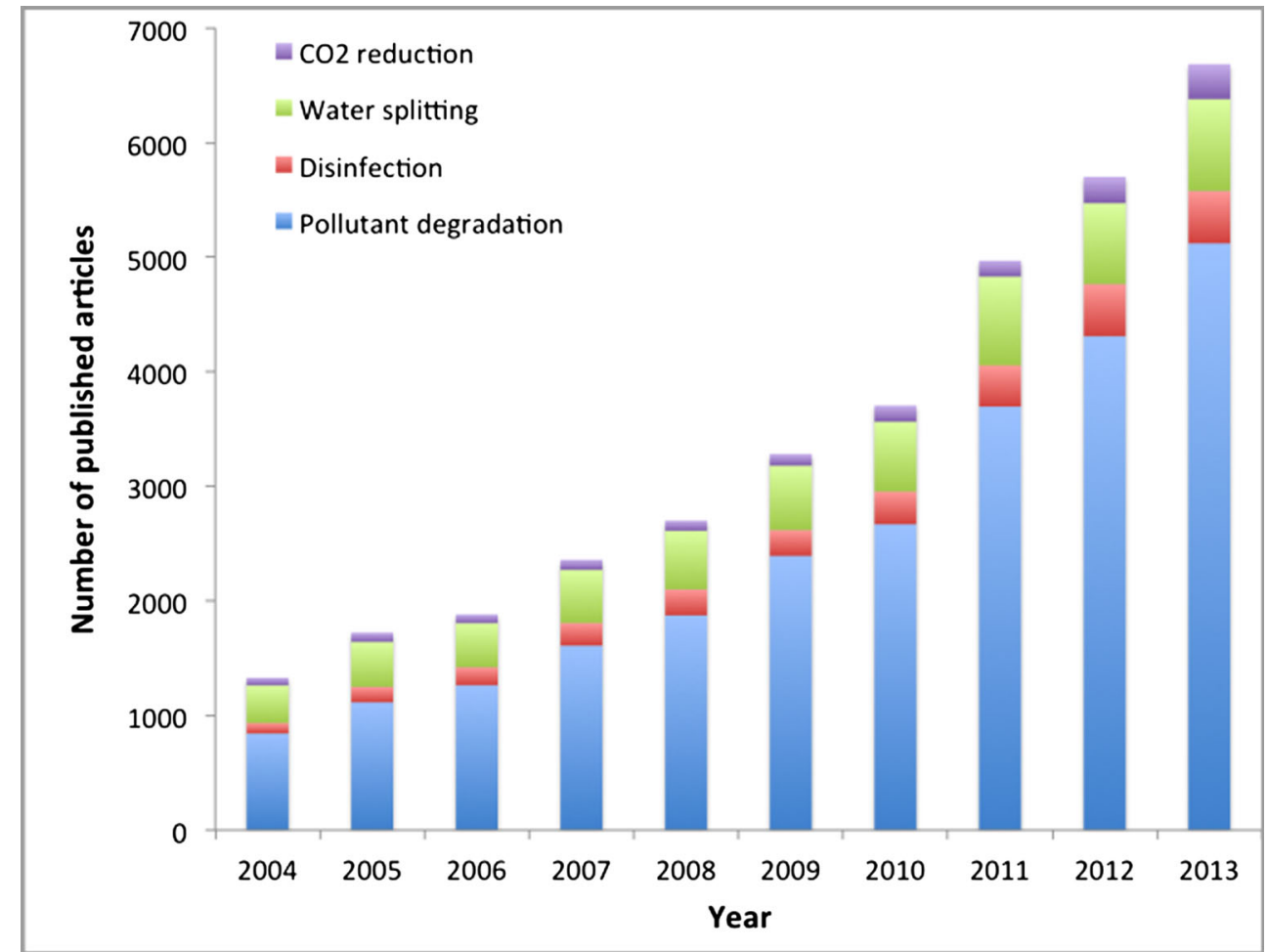

and tiles, paints, paper, concrete and cements, glass, textiles, plastics, etc.).

Along with the increasing concern about the eventual depletion of fossil fuels and the emission of greenhouse gases, the applications of heterogeneous photocatalysis to energy-related processes have been increasing in the last decades. In this context, photocatalysis renders the possibility not only to obtain fuels using the paradigmatic renewable energy of the sun but also to chemically store this intermittent energy source. The apparently simple but actually very energetically demanding process of water splitting to yield hydrogen and oxygen has gathered a great deal of attention from an energetic point of view because it holds the promise of obtaining a clean fuel and energy vector like $\mathrm{H}_{2}$ from an ubiquitous and cheap resource like water. The use of a photocatalyst makes the process feasible with photons within the solar spectrum. In another approach, as the concentration of $\mathrm{CO}_{2}$ in the atmosphere continuously increases due to anthropogenic sources, mainly derived from the use of fossil fuels and deforestation, the possibility of converting the thermodynamically stable $\mathrm{CO}_{2}$ molecule into useful compounds is becoming a challenging goal and a research priority in environmental catalysis. By means of photocatalysis, this process can proceed at mild conditions, mimicking the photosynthesis in green plants, although with the production of simpler compounds such as methanol instead of sugars.

Last but not least, the application of heterogeneous photocatalysis as a promising green method for performing selective organic transformations is gaining importance in the last years.

Up to now, the most used semiconductor for photocatalytic depollution and active surface design is titanium dioxide $\left(\mathrm{TiO}_{2}\right)$. According to Research and Markets (http://www.researchandmarkets.com/research/ 802a1f/commercial_applica), the commercial potential of nanoscale $\mathrm{TiO}_{2}$ coatings is massive, including medical applications, architectural (particularly cultural heritage purposes), construction (façade and indoor paints and coatings, wall papers, tiles, consumer goods, etc.), automotive and food industries (cleaner technologies, non-fogging glass and mirrors, product safety), textile and glass industry, and environmental protection (water and air purification and disinfection). The largest part, accounting for $75 \%$ of the global market and acting in the coming years as a driving force for the implementation of the technology, will still worldwide concern the construction sector with both outdoor and indoor products (self-cleaning glass and tiles, windows, textiles, 


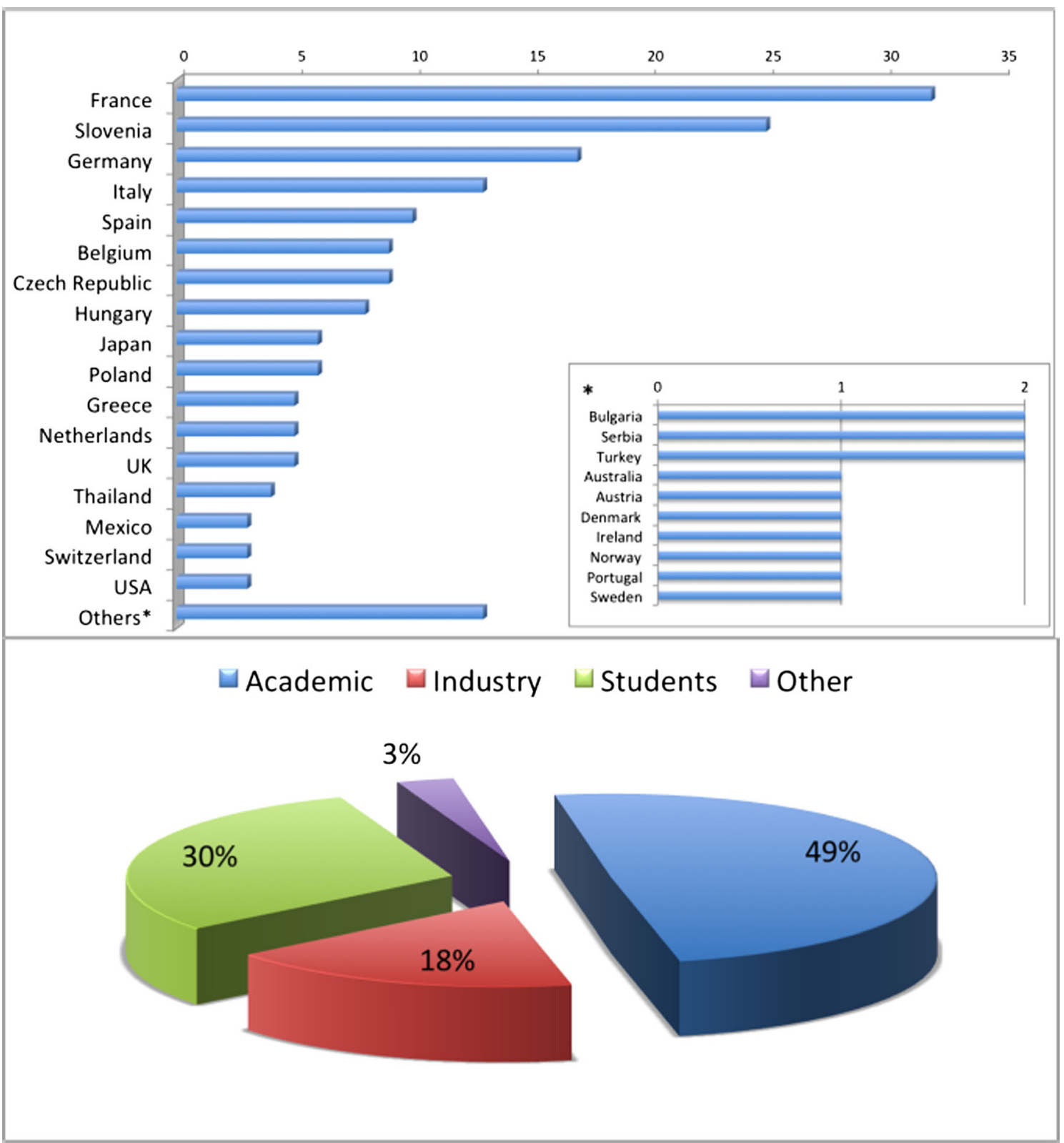

Fig. 2 JEP2013 attendees by country and sector

cement, concrete, paintings, anti-microbial coatings, anti-fogging mirrors...), among which one of the most significant current and future market is the de- $\mathrm{NO}_{\mathrm{x}}$ outdoor air treatment (building walls, tunnels, roofs, roads,...). The 2010 (BCC Research 2010) study analysed that the estimated global market has strongly increased starting from 2005 to reach $€ 1,500$ million in 2015. Asia is actually the major market with $60 \%$ of the global revenues, but the European market will comfort its second ranking, with a yearly $20 \%$ progression, well ahead of the American market.
Within this context, the European Photocatalysis Federation (EPF, http://www.photocatalysis-federation.eu/, born in 2010) with more than 100 European members (companies, universities, technical centres and research laboratories) aimed at the development of the photocatalysis market in Europe. EPF is currently organized in four local committees, namely the German Federation for Applied Photocatalysis (FAP), the Iberian Association for Photocatalysis (AIF), the French Photocatalysis Federation (FFP) and the Czech Association for Application of Photocatalysis (ČSAF). 
One of the main issues for market development is related to standardization. Besides the already published International Standard Organisation (ISO) standards on photocatalytic fine ceramics and advanced technical ceramics, several new ones are under progress with the TC206 WG9 ISO working group (Mills et al. 2012). In Europe, the CEN TC386 has been working for several years on European standards, and eight working groups are going to propose European standards (WG1: terminology; WG2: air purification; WG3: water purification; WG4: self-cleaning; WG6: light sources; WG7: new technologies and other important issues; WG8: microbiological effects). Many companies and academics, as well as EPF, are involved in the definition of these standards, and the first ones are expected in 2014. The next step will then be the certification of commercial products, and relationship with the Photocatalysis Industry Association of Japan (PIAJ) will be helpful in this respect, regarding their experience in those topics. In this context, it is worth advertising for the next international conference on Photocatalysis, Standardisation and Certification Assisting Commercialization (PSCC) which be held in Prague, on the 3rd and 4th of September 2014 on behalf of CSAF, EPF and PIAJ.

After the two first European conferences on photocatalysis (Journées Européenenes de la Photocatalyse, JEP2009 and JEP2011) organized in Bordeaux (France), the last one JEP2013 was organized in Portorož (Slovenia) in collaboration with the University of Nova Gorica. JEP2013 gathered 176 attendees from 27 different countries, summarized in Fig. 2. Several exhibitors illustrated the wide scope of photocatalysis, among which Kronos $\left(\mathrm{TiO}_{2}\right.$ supplier), BMES (developing a new photocatalytic textile) and Omicron (new LED devices) were present, together with some representatives of European companies and PIAJ.

This special issue presents 13 full articles chosen from a number of high-quality presentations at JEP2013. Two papers are devoted to the application of photocatalysis to construction materials. An efficient experimental setup for de-NOx properties evaluation of construction materials is described in the paper by $\mathrm{S}$. Suárez and her colleagues from CIEMAT, Spain. Realistic outdoor conditions, such as changes in $\mathrm{NO}_{\mathrm{x}}$ concentration, irradiance, temperature and relative humidity are taken into account. Façade paints coated by $\mathrm{TiO}_{2} /$ $\mathrm{Zn}-\mathrm{Al}$ with antifungal efficiency were investigated in the group of J. Ranogajec, Serbia. A relatively fast method to monitor antifungal activity of the commercial façade paints covered with photocatalytic coating is proposed. Antimicrobial effects of photocatalysis are also considered in the article by S. Tallósy, Hungary. Silver modified $\mathrm{TiO}_{2}$ and $\mathrm{ZnO}$ plasmonic photocatalysts in polymer thin films proved to be effective against various pathogen microorganisms under visible light.

Visible-light driven processes inspired some other contributions in this issue as well. Regarding organic synthesis, researchers from the group of G. Palmisano in Italy performed oxidation of gaseous aliphatic alcohols to carbonyls via $\mathrm{TiO}_{2}$ sensitized by a perylene derivative. In the study of $\mathrm{Yu}$ et al., the Netherlands, a computational fluid dynamics room model is built to describe the indoor air flow, light distribution and degradation of an indoor inorganic model pollutant by photocatalytic oxidation. The ability of bismuth vanadate, as a photocatalyst by itself or as titania sensitizer, to mineralize gaseous pollutants (VOCs) is explored in the work by F. Fresno and his Slovenian and Italian colleagues. Another two papers in this issue deal with photocatalytic degradation of VOCs. Influence of inlet air parameters on mineralization of VOCs in air conditioning installations is described by Spanish authors E. Jiménez-Relinque and M. Castellote, while the group of A. Bouzaza et al. from France presents VOCs elimination by $\mathrm{UV} / \mathrm{TiO}_{2}$ photocatalysis in three different reactors with successful scaling up the process.

Other papers in the special issue belong to water treatment by photocatalysis, which can be efficient in removing low concentrations of otherwise stable and persistent pollutants. Effective photocatalytic degradation of pesticides using decatungstate intercalated macroporous layered double hydroxides is reported by M. Sarakha and co-authors from France and Portugal. Pharmaceuticals, another type of emerging water pollutants, are the subject of two studies. The group of S. Zorita, Spain, describes synergy effect of photodegradation and adsorption of pharmaceuticals on a suspended mixture of titania and activated carbon. On the other hand, ciprofloxacin effluents are efficiently degraded by continuous flow photo-Fenton process as described in the paper by A.M.T. Silva et al., Portugal. Surfactants, again another type of emerging water contaminants, are effectively decomposed by $\mathrm{TiO}_{2}$-photocatalysis combined with ozonation in the work by O. Horváth et al., Hungary. Lastly, in the contribution of U. Lavrenčič Štangar and her co-workers, Slovenia, stable and highly active coatings on glass are presented, which are obtained by incorporation of commercial titania powders in a sol-gel matrix. 
Authors and reviewers are greatly acknowledged for their contributions. Dr. Philippe Garrigues, ESPR Editor-in-Chief, is sincerely thanked for the kind invitation to publish this issue.

\section{References}

BCC Research (2010) http://www.bccresearch.com/pressroom/avm/ global-photocatalysts-market-worth-\$1.7-billion-2014

Mills A, Hill C, Robertson PKJ (2012) J Photochem Photobiol A Chem 237:7-23

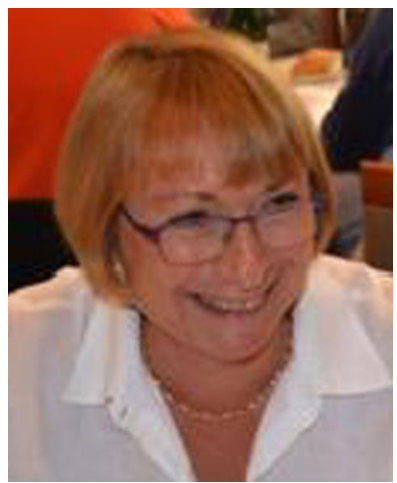

Sylvie Lacombe is CNRS research director in the Institute for Analytical Sciences and Physico-Chemistry for Environment and Materials (IPREM), a mixed CNRSUPPA (University of Pau and Pays de l'Adour) team. She has been working for 20 years in the photochemistry and photocatalysis field and was interested with sol-gel chemistry for the synthesis of photosensitizers containing hybrid materials or modified $\mathrm{TiO}_{2}$ and with their application for the oxygenation/mineralization of organic compounds. Current research topics are devoted to reaction mechanisms in heterogeneous photocatalytic/photosensitized reactions. She is also interested with normalization in photocatalysis.

She is in charge of the photochemistry group in Pau, and she is vice-president of the European Photocatalysis Federation http:// www.photocatalysis-federation.eu/.

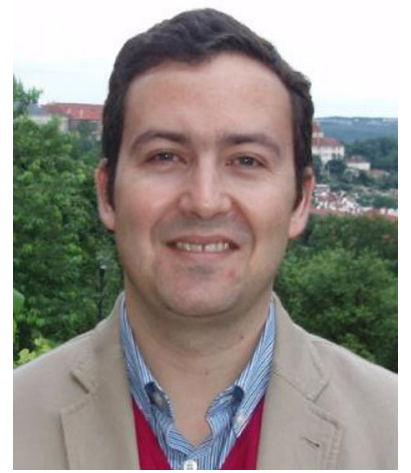

Fernando Fresno is currently a Research Associate in the Laboratory for Environmental Research and Assistant Professor in the School of Environmental Sciences at the University of Nova Gorica, Slovenia. He has conducted postdoctoral work at ICP-CSIC and CIEMAT, Spain. He got his Ph.D. in Chemistry in 2006 from the Autónoma University of Madrid (UAM), working at ICP-CSIC and the Inorganic Chemistry Department of UAM. He has worked as a visiting researcher at the Universities of Niigata (Japan) and Aberdeen (UK) and IRCELYON (France). His research interests focus on photocatalytic processes with emphasis on the synthesis of new, more efficient photocatalytic materials.

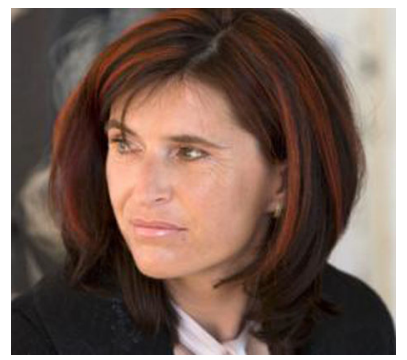

Urška Lavrenčič Štangar is a senior researcher in the Laboratory for Environmental Research at the University of Nova Gorica, Slovenia, and dean of its School of Environmental Sciences. After getting her Ph.D. in 1996, she continued the research at the National Institute of Chemistry, Ljubljana, on inorganic thin films and hybrid organic-inorganic gels for their application in devices using sustainable energy. She joined the University of Nova Gorica in 2005 , focusing her research activities in the field of photocatalysis. Her work includes the preparation and characterization of materials in thin-film and powder form and their role in environmental cleaning. She is currently involved in several research projects on photocatalysis and is an academic committee member of the European Photocatalysis Federation. 